\title{
Modeling Aging and Yielding of Complex Fluids: Application to an Industrial Material
}

\author{
A. Ragouilliaux ${ }^{1,2}$, P. Coussot ${ }^{2}$, T. Palermo ${ }^{1}$ and B. Herzhaff ${ }^{*}$ \\ 1 Institut français du pétrole, IFP, 1-4 avenue de Bois-Préau, 92852 Rueil-Malmaison - France \\ 2 Université Paris-Est, Institut Navier, LMSGC, 2 allée Kepler, 77420 Champs - France \\ e-mail: alexandre.ragouilliaux@lcpc.fr - philippe.coussot@lcpc.fr - thierry.palermo@ifp.fr - benjamin.herzhaft@ifp.fr \\ * Corresponding author
}

\begin{abstract}
Résumé - Modélisation du vieillissement et de l'écoulement de fluides complexes : application à un matériau industriel - Les fluides complexes naturels ou rencontrés dans de nombreux procédés industriels sont souvent composés de plusieurs phases formant des émulsions, mousses ou suspensions colloïdales plus ou moins concentrées. La plupart de ces fluides peuvent être décrits de manière générale comme des «systèmes vitreux mous» présentant une transition solide-liquide lorsqu'une contrainte suffisante leur est appliquée. La description physique de cette transition est essentielle pour comprendre la dynamique de l'arrêt dans des processus naturels (avalanches de neige, glissements de terrain, etc.) ou des procédés sous faibles sollicitations mécaniques impliquant certains matériaux industriels comme les bétons auto-plaçant, les colles, les crèmes cosmétiques et certains fluides pétroliers. Nous nous intéressons ici au lien entre la microstructure d'un fluide complexe, et son comportement rhéologique macroscopique, particulièrement lors de la transition solide-liquide caractéristique de ces systèmes. En couplant la rhéométrie conventionnelle qui nous donne les propriétés macroscopiques du matériau, et la vélocimétrie IRM pour avoir accès aux propriétés locales, nous pouvons identifier les origines structurelles des principales propriétés rhéologiques telles que le seuil de contrainte, le vieillissement au repos et la bifurcation de viscosité en régime liquide. En particulier nous montrons que l'arrêt progressif de l'écoulement induit par la croissance d'agrégats sous une contrainte critique explique certaines particularités des courbes d'écoulement. Nous montrons par la suite comment le comportement transitoire et stationnaire du fluide peut être décrit par un modèle simple de thixotropie faisant intervenir un paramètre structurel dépendant du temps et du cisaillement. Une application pratique de ce modèle est proposée, montrant comment les paramètres du modèle peuvent être déduits d'expérimentations simples, et comment le modèle peut être utilisé pour prédire les conditions de redémarrage d'un fluide à l'arrêt dans une conduite. Ce travail permet de proposer des éléments de modélisation microscopique du comportement thixotrope de ces fluides complexes en relation avec leur structure, et montre l'application pratique de cette démarche de modélisation.
\end{abstract}

\footnotetext{
Abstract - Modeling Aging and Yielding of Complex Fluids: Application to an Industrial Material Complex fluids either natural or encountered in numerous industrial processes are often composed of several phases constituting emulsions, suspensions, foams or other colloidal dispersions. Many of these complex fluids may be described in a general manner as "soft-jammed systems" which have the ability to undergo a solid-liquid transition when submitted to a sufficient stress. The description of this transition from a solid state to a flowing situation is essential to understand for example the dynamic of flow stoppage or restart of natural processes (snow avalanches, ground sliding, etc.) or of industrial processes (self placement concrete, glues, cosmetic formulations, mud circulation, flow assurance, etc.).
} 
In this study, we have interest in the link between the microstructure of a complex fluid and its macroscopic rheological behavior, especially regarding the solid liquid transition characteristic of these systems. By coupling conventional rheometry giving macroscopic properties, and IRM velocimetry giving access to local properties, we can identify the structural origin of the major rheological properties as the yield stress, the aging at rest and the viscosity bifurcation in the liquid regime. We show that the progressive stoppage of the material, induced by the growing of aggregates under a critical stress explains some peculiar characteristics of the flow curves. We show then how the transient and stationary behavior of the fluid may be described by a unique thixotropic model involving a structural time and shear dependant parameter. A practical application of this model is proposed, showing how the parameters of the model may be deduced from simple experiments, and how the model may be used to predict restart conditions after rest for a fluid flowing in a pipe. This work allows to propose elements of microscopic modeling of the thixotropy of these systems in relation with their structure, and show the applicability of this modeling work to practical situations.

\section{INTRODUCTION}

A lot of industrial processes, especially in the energy industry, implies the flow (imposed or undergone) of complex fluids. Well drilling (drilling muds), stimulation operations (fracturation fluids), enhanced oil recovery processes (polymers, microemulsions), effluents production (emulsions, production water), or event optimization of well sealing for storage purpose (cement materials), all these operations need to control the flow properties of these complex fluids, in various conditions. These fluids are often constituted of several phases (usually a continuous phase with one or several dispersed phases) which form emulsions, suspensions, foams or other colloidal dispersions. Many of these complex fluids may be described in a general manner as "soft-jammed systems" which have the ability to undergo a solid-liquid transition when submitted to a sufficient stress. The description of this transition from a solid state to a flowing situation is essential to understand for example the dynamic of flow stoppage or restart of natural processes (snow avalanches, ground sliding, etc.) or of industrial processes (mud circulation, flow assurance, etc.). Their yielding and aging properties originate from the physical specificities of these systems: they are composed of a great number of small elements in colloidal interaction in the liquid; the elements are jammed, so that the fluid exhibits a yield stress that must be overcome in order to unjam the structure and induce flow; moreover a great number of such elements is an out-of-equilibrium system which evolves under the action of thermal agitation, it ages, so that the fluid exhibits a time-dependent viscosity, it is thixotropic.

As an example of this type of jammed system, we present in the first part of this paper, a detailed study of an industrial complex fluid which is a drilling mud specially formulated for ultra deep offshore conditions and inclined or horizontal well. This complex fluid is an invert emulsion of brine into an oil phase stabilized by surfactant. Various additives are added as organophilic clay, solids and other additives. In order to satisfy the different functions required by the application, these fluids are typically shear thinning and thixotropic and exhibit an apparent yield stress. Their internal structure is generally complex and is liable to evolve with flow conditions. In particular, it has been shown that the flow curve of a typical drilling mud exhibits an abrupt slope change below a critical shear rate (Herzhaft et al., 2003), an effect which might significantly affect the low velocity flow characteristics in pipes. The physical origin of the structure and the structuration kinetics are not described for such systems, and are very important to estimate for example the necessary pressure to restart the flow of the fluid after a time of rest. We describe here the flow curve properties, and the solid liquid transition using classical rheometry and MRI velocimetry giving access to the detailed velocity profiles of the sheared fluid.

We then show in the second part how the thixotropic and shear properties of this kind of jammed fluid could be described with a unique model, with a reduced number of physical parameters that could be determined with simple experiments. A practical application of this model is shown through the calculation of the necessary pressure to restart the flow in a pipe after a time of rest.

Invert emulsion mixed with organoclay suspension may exhibit the behavior of a concentrated emulsion or that of a clay suspension with a significant particles concentration.

Concentrated emulsions classically exhibit a yield stress shear thinning fluid behavior, the value of the yield stress being a function of the volume fraction and the dimension of the droplets. Flocculation and rearrangement of the droplets under shear induce shear thinning behavior. Mason et al. $(1995,1996)$ have shown that above a certain critical volume fraction, a yield stress appears and scales with the Laplace pressure. They show also that above around $70 \%$ of volume fraction, inhomogeneous flows appear throughout the sheared sample.

Velocity profile measurement by NMR techniques have been employed on concentrated emulsions by Hollingsworth (2004) who observed wall slip phenomena. More recently, Becu et al. $(2004,2006)$ by using ultra sound velocity measurement show the occurrence of wall slip and shear 
banding evolving in time in a Couette geometry when the emulsions present "adhesive" interactions. The effects of surfactant and droplets deformation under shear have also been studied in order to understand the origin of the behavior of these systems (see for example the review of Barnes, 1994).

In parallel the rheological behavior of clay-water suspensions has been widely studied (see for example the review in Coussot, 1997). Different behaviors have been documented like shear banding or fracturing with complex time dependency (Coussot, 2005). In the complex systems studied here, the colloidal particles are suspended in oil and participate to the thixitropic behavior of the system, but their detailed rheological behavior has not been described yet.

\section{MATERIALS AND PROCEDURES}

\subsection{Material}

The industrial material studied is basically a concentrated water-in-oil emulsion with various additives such as organophilic polymers, organophilic clay, solids, etc (see Tab. 1). Among these additives the surfactants and colloidal particles (Yan et al., 2001) stabilize the emulsion while coarse solid particles increase the mud density.

The fluid was prepared by successively adding the components in a Silverson ${ }^{\circledR}$ L4RT mixer at $6000 \mathrm{rpm}$. Then it was put in a roller oven under relatively high pressure (10 bars) and temperature $\left(80^{\circ} \mathrm{C}\right)$ during 16 hours in order to accelerate its aging under conditions similar to those prevailing in well exploitation. After such a preparation the material appeared very stable, i.e. its behavior and structure did not evolve significantly over several weeks and no significant sedimentation occurred over several days. The droplet size as it appeared from microscopy (Leica phase contrast microscope) was uniform with an average diameter of $2 \mu \mathrm{m}$.

\subsection{Procedures}

For conventional rheometry we used stress controlled rheometers, Haake RS 150 and Bohlin C-VOR 200, equipped respectively with a rough Couette cell (inner radius: $r_{1}=19 \mathrm{~mm}$; outer radius: $r_{2}=21.5 \mathrm{~mm}$; height: $h=55 \mathrm{~mm}$; roughness: $200 \mu \mathrm{m}$ ) and a vane tool as inner cylinder insert in a rough Couette cell (inner radius, $r_{\mathrm{i}}=12.5 \mathrm{~mm}$; outer radius, $r_{\mathrm{o}}=18 \mathrm{~mm}$; height, $h_{v}=45 \mathrm{~mm}$; roughness: $180 \mu \mathrm{m}$ ). In order to obtain the same initial state, the sample was first mixed in the Silverson apparatus at $6000 \mathrm{rpm}$ during 15 minutes then set up in the rheometer, presheared at $1000 \mathrm{~s}^{-1}$ during 1 minute and left at rest during 10 secondes. Afterwards we imposed a logarithmic increasing-decreasing stress ramp composed of 30 stress steps, the duration of each
TABLE 1

Constituents, time of shear and introduction order for the industrial material

\begin{tabular}{|c|c|c|c|}
\hline Products & $\begin{array}{l}\text { Time } \\
\text { of shear } \\
\text { (min) }\end{array}$ & $\begin{array}{l}\text { Order for } \\
\text { mixing }\end{array}$ & $\begin{array}{l}\text { Quantities } \\
\text { (\% of the } \\
\text { total mass) }\end{array}$ \\
\hline HDF 2000 (dearomatized oil)** & 5 & 1 & 40.2 \\
\hline Versatrol $^{T M}$ (filtrate reducer)* & 5 & 2 & 0.53 \\
\hline Versamul $^{T M}$ (emulsifier)* & 5 & 3 & 1.64 \\
\hline Versawet $^{T M}$ (wetting agent)* & 5 & 4 & 0.26 \\
\hline Lime & 5 & 5 & 1.79 \\
\hline$V G 69^{T M}$ (organophilic clay)* & 5 & 6 & 1.35 \\
\hline Versa HRP $P^{T M}$ (viscosifyer)* $^{*}$ & 5 & 7 & 0.37 \\
\hline Brine & 10 & 8 & 28.81 \\
\hline $\mathrm{CaCO}_{3}$ (weighting agent) & 20 & 9 & 23.25 \\
\hline
\end{tabular}

* MI formulation, ** Total solvent.

stress step being 45 secondes. In another experiment, we imposed various fixed stress levels during one hour, after having imposed similar preshear and rest between each experiment. The temperature of the sample was maintained at $20^{\circ} \mathrm{C}$ during all tests.

For MRI-rheometry we used a set-up and procedures described in details elsewhere (Raynaud et al., 2002). We used a vertical coaxial cylinder geometry (inner cylinder radius: $r_{1}{ }^{\prime}=40 \mathrm{~mm}$; outer cylinder radius: $r_{2}{ }^{\prime}=60 \mathrm{~mm}$; height: $h_{C}=11 \mathrm{~cm}$; roughness: $\left.200 \mu \mathrm{m}\right)$. The rotation velocity of the inner cylinder $(\Omega)$ was controlled. The room temperature was kept between 20 and $22^{\circ} \mathrm{C}$. The samples were first mixed as above described then inserted in the geometry and left at rest $30 \mathrm{~s}$ before a fixed rotation velocity being imposed to the inner cylinder. Each velocity profile presented here corresponds to the local velocity averaged over eight seconds and the MRI procedure requires to wait two seconds between two successive profiles.

No sedimentation or segregation effects were observed in the sample at the end of conventional and MRI rheometry. Moreover conventional MRI density imaging on our samples did not show any significant density heterogeneity. As a consequence, owing to the small droplet size and the large solid fraction of droplets interacting via clay particles, it is likely that the concentration of droplets remains homogeneous during all our tests.

\section{RHEOLOGY OF THE INDUSTRIAL COMPLEX FLUID}

\subsection{Flow Curve}

Figure 1 represents the flow curve obtained using the increasing/decreasing stress ramp procedure described in 


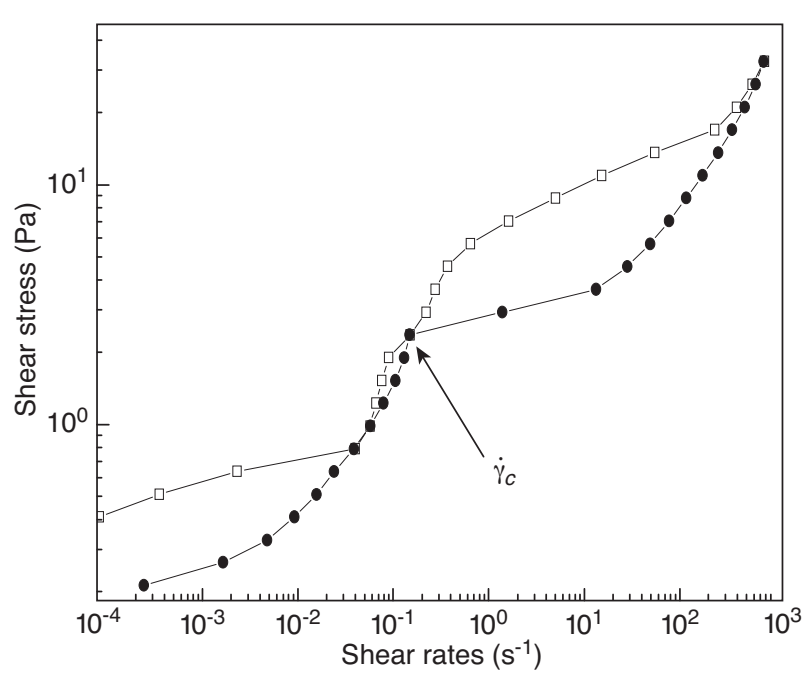

Figure 1

Flow curve of the industrial complex fluid obtained with an increasing (empty squares) and decreasing stress ramp (full circles). The sample is initially mixed in the Silverson apparatus at $6000 \mathrm{rpm}$ during $15 \mathrm{~min}$ then set up in the rheometer, presheared at $1000 \mathrm{~s}^{-1}$ during $1 \mathrm{~min}$ and left at rest during $10 \mathrm{~s}$. The duration of each stress step is $45 \mathrm{~s}$.

Section 1.2. A large hysteresis in the increasing/decreasing curves indicates the strong thixotropy of the system. The decreasing part which should correspond to the steady flow of the system, presents an abrupt slope change at some critical shear rate, with the stress decreasing strongly towards low shear rates, instead of reaching a plateau characteristic of yield stress fluids. Rough surfaces were used for the geometries, eliminating in principle significant wall slip as the reason for this peculiar flow curves. This slope change allows us to define two critical values, $\tau_{\mathrm{C}}$ and $\dot{\gamma}_{\mathrm{C}}$ characteristic of the system.

\subsection{Solid Liquid Transition}

The transition zone around the critical stress value was investigated by carrying out systematic creep test over long time, following the procedure described in Section 1.2. The results are presented in terms of the shear rate as a function of time (see Fig. 2). Two periods may be defined: during about $200 \mathrm{~s}$, the shear rate slightly decreases as the stress level decreases but is approximately constant with time; after that first period, the shear rate starts to decrease in various extents according to the stress level. Two regimes can be distinguished in this second period: beyond a critical stress (between 2.8 and $2.85 \mathrm{~Pa}$ ) the shear rate reaches different, finite values decreasing as the stress decreases; below this critical stress the shear rate is several decades smaller and reaches a value, independent of the stress but still decreasing after one hour of flow. This abrupt transition at the critical stress is the signature of a very strong viscosity bifurcation (Coussot et al., 2002a, b). In the first regime (above the critical stress) the material flows steadily, with classical shear thinning characteristics, but in the second regime (beyond the critical stress), the material tends to stop progressively reaching very high values of viscosities increasing with time. This transition indicates that the material is not able to flow at a shear rate lower than a critical value $\left(0.57 \mathrm{~s}^{-1}\right.$ here $)$ corresponding to the lowest shear rate of the first regime. A striking point is that this transition takes place for a very reduced stress level difference: a $0.05 \mathrm{~Pa}$ stress difference is sufficient for the material to pass from a steady flow at $0.6 \mathrm{~s}^{-1}$, to a quasi solid state with a residual flow around $10^{-6} \mathrm{~s}^{-1}$. The time for this transition to occur is also to be noticed: almost $200 \mathrm{~s}$ of quasi Newtonian flow regime is recorded before the bifurcation takes place.

The critical shear rate and shear stress values are consistent with the slope change of the flow curve obtained with stress ramps. It seems therefore that the part of the flow curve below the critical stress can be identified to this quasi solid regime where no steady flow is reached, and that the shear recorded in this regime correspond to discontinuities of structure (shear banding) of the sample. Local rheometry experiments are necessary to investigate this feature.

From these experiments, it is possible to extract the equilibrium flow curve, at least for the part of the flow curve above the critical shear stress. Figure 3 represents this equilibrium flow curve obtained from creep tests, together with flow curves obtained with increasing/decreasing stress ramp.

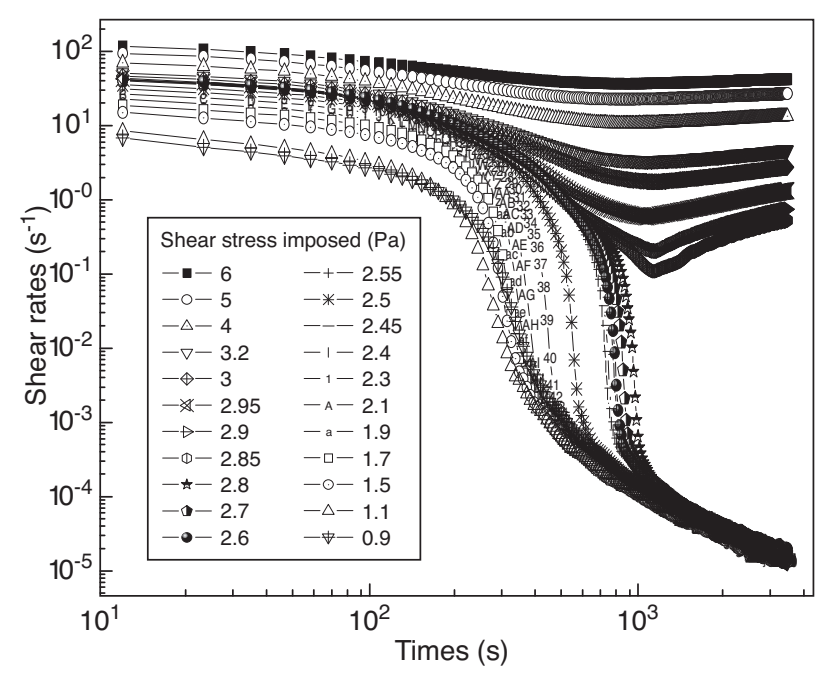

Figure 2

Creep test from conventional rheometry expressed as the shear rates evolution in time for different shear stress imposed between 0.9 and $6 \mathrm{~Pa}$. 


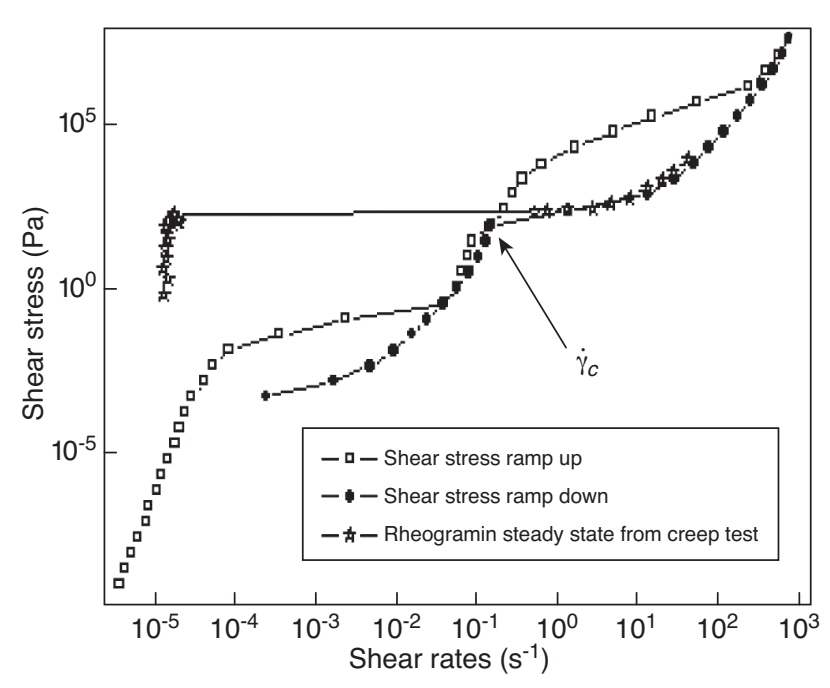

Figure 3

Equilibrium flow curve extracted from creep tests, together with flow curves obtained with increasing/decreasing stress ramp.

\subsection{Local Rheometry}

The stress ramp experiments suggest that it is impossible for the material to reach steady shear rates below a certain critical value. This could explain the flow curves obtained and rises the question of the homogeneity of the flow in the gap of the rheometer. Local rheometry using MRI was therefore carried out in order to clarify these questions. The procedure is described in Section 1.2. We measure successive tangential velocity profiles into the rheometer gap as a function of time. Figure 4 gathers the profiles obtained.

We observe again two time periods. Initially, during about $100 \mathrm{~s}$ of flow, the entire gap is sheared and the velocity profile doesn't vary with time. We observe a continuous profile which is consistent with a power law fluid sheared in the rheometer gap. It is possible to deduce values of index of rheofluidification from these curves (see Ragouilliaux, 2006 for more details).

After this first period, we observe an evolution of the velocity profile which progressively splits in two parts before reaching a steady state shape. Adjacent to the outer non moving cylinder, a zone of almost constant velocity progressively tending to zero appears while a zone close to the inner rotating cylinder keeps its power law fluid profile on a decreasing radius. In Figure 5, we represent the velocity evolution with time along the gap (each profile corresponds to a radius inside the gap). This profile evolution indicates a viscosity bifurcation consistent with the observations in creep tests in conventional rheometry. We can define a critical radius in the rheometer gap delimiting the two part of the steady state velocity profile. As for radii lower than this critical radius, the material flows continuously reaching a constant velocity; for radii superior to this critical radius, after a transient period of constant velocity, the tangential velocity abruptly decreases to zero (the viscosity continuously increases to very large values), and the material finally stops flowing. There is therefore an unsheared region in the steady state profile which develops progressively during a transient period. The size of this unsheared region in the steady state increases as the rotation velocity decreases (see Ragouilliaux, 2006 for more details).
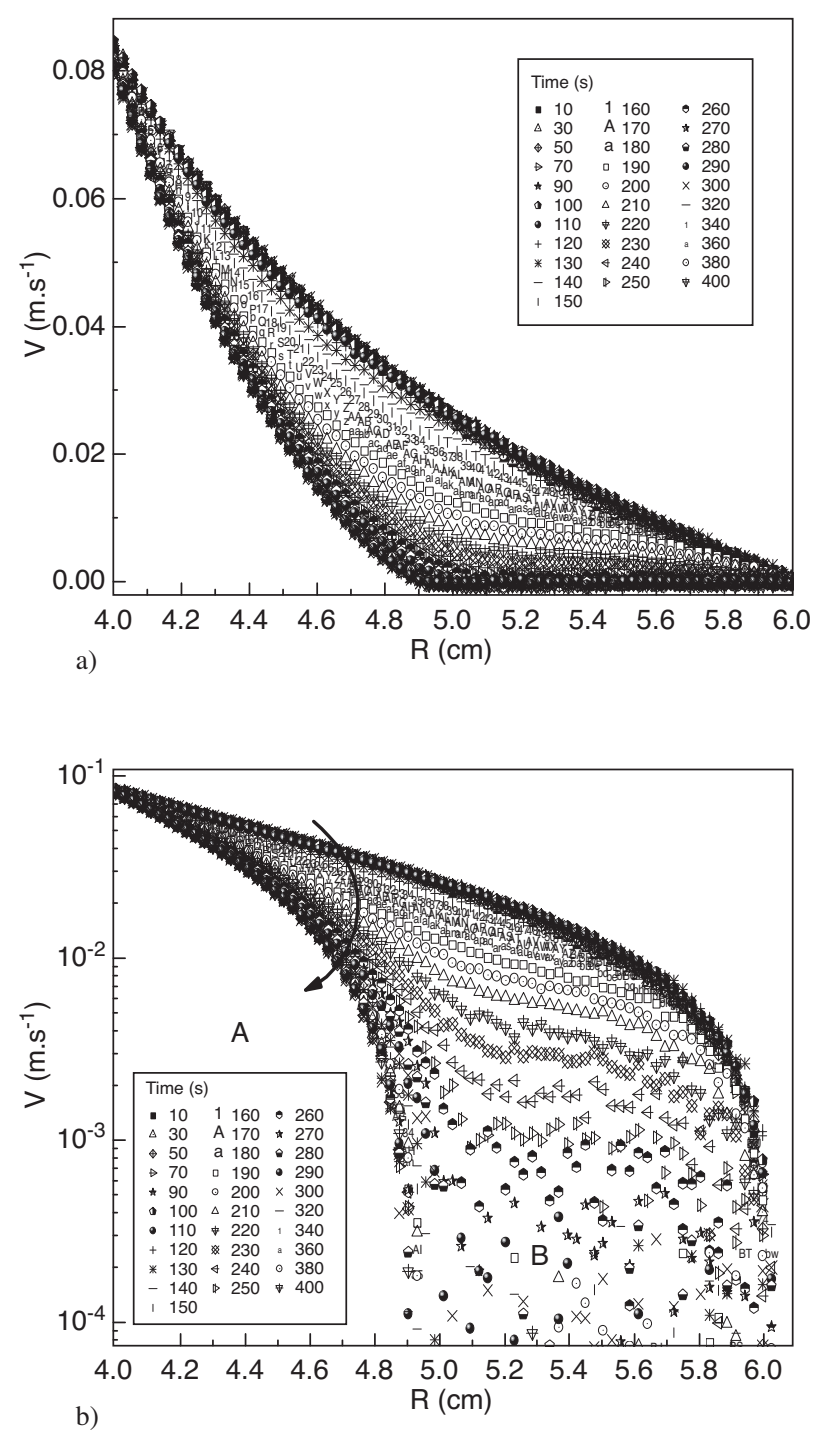

Figure 4

Velocity profiles measured during $400 \mathrm{~s}$ for a rotation velocity imposed of $20 \mathrm{rpm}$ : a) linear representation and b) semi-logarithmic representation. 


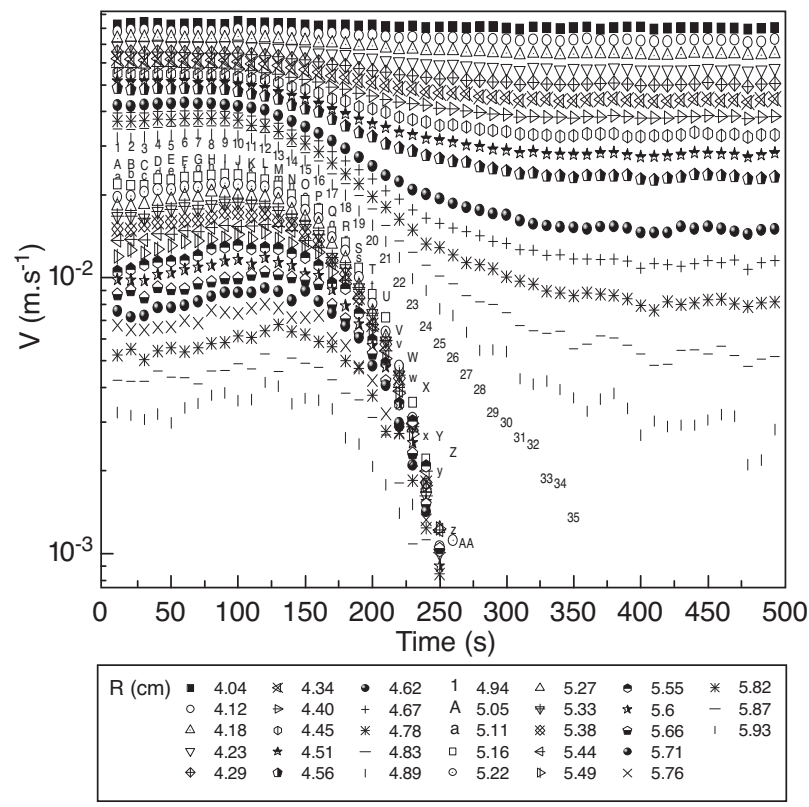

Figure 5

Viscosity bifurcation in the gap of the MRI Couette cell.

These local rheometry observations are consistent with the results obtained in classical rheometry. They show that the material present a critical shear stress and that when pre sheared, the material will flow under stress first like a power law fluid during a very long transient period. Then, if the applied stress value is higher than the critical shear stress, the material will reach a steady flow behavior at a shear rate larger than a minimum value. When the applied stress is lower than the critical shear stress, the material will progressively increase its viscosity upon complete stoppage. Let's notice that the duration of the transition period depends on the applied stress value and can reach very large values (around $200 \mathrm{~s}$ ).

This behavior is the signature of a progressive aging of the material opposed to a shear rejuvenation imposed by the flow characteristic. The nature of the aging of this material has been investigated through systematic experiments on model fluids i.e. pure emulsions and clays loaded emulsions. Details of this investigation can be found in an other publication (Ragouilliaux et al., 2007). It has in particular been shown that the thixotropy of the loaded emulsion is due to a progressive aggregation of droplets via colloidal particle links, which tends to increase the viscosity of the material which finally stops. During stoppage the shear rate exhibits a specific evolution which is consistent with the predictions of a model assuming a progressive aggregation of droplets in time.

\section{PRACTICAL APPLICATION OF A THIXOTROPIC MODEL}

As mentioned, the peculiar behavior of the fluid can be explained by an intrinsic competition between natural aging (progressive aggregation of droplets) and shear rejuvenation imposed by the flow. This competition implies the existence of a critical shear value as observed through the rheological and local velocimetry experiments: above this critical value, shear rejuvenation is dominant and the material reaches a stable flow regime, below this critical value, aging is dominant with time, and the material tends to final stoppage. The experimentally determined transient period could therefore be explained by the different characteristic kinetics of aging and shear rejuvenation. These results show that it is natural to seek a description of yielding and thixotropy from a single rheological model including time evolution, and not to separate the description of "stable shear properties" from "gel building properties". Such a model should therefore incorporate a kinetic equation rendering the competition between aging and rejuvenation.

Our objective in this part is to propose such a unified model, relying on relevant physical mechanisms, and see how the model may be used practically to describe field needed properties. In particular, simple experiments are proposed to derive the needed model parameters.

\subsection{A Simple Model for a Thixotropic Yield Stress Fluid}

From a general point of view we expect a thixotropic fluid like the complex emulsion studied in this work to have a constitutive equation such that in simple shear the local shear stress at a given time $t$ depends not only on the local current shear, but also on the local flow history (which can be described by the history of the shear rate) and a state of reference of the material (typically its state at the initial time). A practical means often used in rheology consists in considering that the current stress in fact only depends on the current shear rate through a function which also depends on the "state of structure" (Cheng and Evans, 1965; De Kee and Chan Man Fong, 1994). This state of structure is often described by a single variable $\lambda$ generally referred to as the structure parameter, and which is a function of flow history. It is for example related to the number of links between particles in a flocculating system or to the average depth of the potential wells of particles in a non-flocculating suspension. In our case, $\lambda$ should be related to the number of clayed links created between droplets. The stress dependence on flow history is now accounted for via a kinetic equation for $\lambda$ rendering the competition between aging (construction of links) and rejuvenation (destruction of links), the solution of which depends on flow history. Various models of this type have been proposed (Coussot et al., 1993; Chan Man 
Fong et al., 1996; Mujumdar et al., 2001) but, in the absence of systematic studies of their field of validity none of them can be considered as more appropriate than the others. Here we follow an approach already presented in several recent papers (Coussot et al., 2002a; Roussel et al., 2004) for pasty fluids such as bentonite suspensions, which consists to use the simplest thixotropy model capable to reproduce the main qualitative trends observed with such fluids.

In this model, in simple shear, the apparent viscosity $\mu=\sigma / \dot{\gamma}$ of the fluid, in which $\sigma$ and $\dot{\gamma}$ are the shear stress and shear rate amplitudes, is a simple function of the structure parameter at the current time $t$ :

$$
\mu=\frac{\sigma}{\dot{\gamma}}=\mu_{0}\left(1+\lambda^{n}\right)
$$

in which $\mu_{0}$ and $n$ are two fluid parameters. Equation (1) simply predicts that the apparent viscosity increases with the structure parameter, i.e. with the number of links between components. Now the critical point is the equation describing the evolution of $\lambda$ with time. We assume that this "kinetic equation" takes a form often used in this field:

$$
\frac{d \lambda}{d t}=\frac{1}{\theta}-\alpha \lambda \dot{\gamma}
$$

The physical meaning of (2) is as follows: the rate of change of the structure parameter (left hand-side) is equal to the difference between a rate of restructuration $(1 / \theta)$ which is assumed to be an intrinsic characteristic of the material, and a rate of destructuration due to flow, which is naturally assumed to be proportional to the local shear rate since the "amount" of destructuration is expected to be proportional to the deformation undergone by the material. At any time, there is a competition between aging (intrinsic property) and shear rejuvenation (flow property), and the equation (2) is the simplest mathematical form to describe this physical process. It is worth noting that more complex functions of $\lambda$ (for example power-law functions) may be used for the two terms of the right hand-side of (2), on the basis of various phenomenological arguments, but in most cases it would be possible, through a change of variable, to get back a set of equations formally analogous to (1)-(2) with now, instead of $\lambda$, a function $\Pi(\lambda)$. In the absence of a clear physical meaning for $\lambda$ such models are strictly equivalent for describing flow characteristics (Coussot, 2005). This suggests that the above set of equations provides a robust phenomenological description of flow characteristics, taking into account the basic physical aspects of thixotropy.

One interest of the above model is that flow simulations do not require the determination of the solid-liquid limit as with other yield stress models: the material always flows everywhere and the (apparently) unyielding regions simply correspond to the parts of the material in which the viscosity tends to infinity as time goes on. Thus, although it does not contain an explicit yield stress, this model predicts flow characteristics apparently similar to those of a yield stress fluid following one of the usual models in this field. This model was shown to well predict qualitative rheological trends of various pasty materials, exhibiting or not significant thixotropic characteristics (Coussot et al., 2002a). In particular when $n>1$ it predicts the viscosity bifurcation effect observed under constant stress values (Coussot et al., 2002b). The quantitative predictions of this model were also successfully compared with local and macroscopic data concerning viscometric flows (Roussel et al., 2003; Ragouilliaux et al., 2006).

\subsection{Steady-State Behavior of the Fluid}

Let us now review the main properties of a fluid exhibiting a behavior as described by Equations (1) and (2). In steady state at a shear rate $\dot{\gamma}_{S}$ the structure parameter reaches the steady value $\lambda_{S}=1 / \alpha \theta \dot{\gamma} \dot{\gamma}_{S}$ and the shear stress writes:

$$
\sigma_{S}=\mu_{0}\left[1+\left(\frac{1}{\alpha \theta \dot{\gamma}_{S}}\right)^{n}\right] \dot{\gamma}_{S}
$$

For $n<1$ the fluid does not exhibit a yield stress, a case without interest here. For $n>1$ the shear stress decreases with increasing $\dot{\gamma}$ for $\dot{\gamma}<\dot{\gamma}_{c}=(n-1)^{1 / n} / \alpha \theta$ and increases otherwise. It may be shown from a linear stability analysis that flows corresponding to the decreasing part are unstable. This implies that in practice shear localization tends to occur for $\dot{\gamma}<\dot{\gamma}_{c}$ so that finally for $n>1$ the apparent behavior of the material is as follows:

- solid below a critical stress: $\sigma<\sigma_{c} \Leftrightarrow \dot{\gamma}=0$;

- purely plastic for small shear rates: $0<\dot{\gamma} \leq \dot{\gamma}_{c} \Leftrightarrow \sigma=\sigma_{c}$;

- liquid for large shear rates: $\dot{\gamma} \geq \dot{\gamma}_{c} \Rightarrow \sigma=\mu_{0}\left[1+(1 / \alpha \theta \dot{\gamma})^{n}\right] \dot{\gamma}$.

Thus the apparent behavior predicted by the model in steady state is close to that of a simple yield stress fluid with an apparent yield stress which can be estimated by:

$$
\sigma_{c}=\sigma_{s}\left(\dot{\gamma}_{c}\right)=\mu_{0}\left(\frac{n}{n-1}\right) \dot{\gamma}_{c}
$$

In Figure 6 is represented the steady state answer of the model for classical values of the parameters.

This behavior is generally that which can be observed from a stress or shear rate ramp after a strong preshear. We therefore obtain in steady state the typical yield stress shearthinning behavior of the industrial fluid under constant shear, although no value of yield stress was assumed a priori.

\subsection{Apparent Yield Stress}

Remark that strictly speaking this model describes the material as if it was always a liquid. The solid regime in fact corresponds to a situation for which $\lambda \rightarrow \infty$ so that the apparent viscosity tends towards infinity. This is a weakness of the model but for a more precise description of the two regimes it would be necessary to introduce additional 


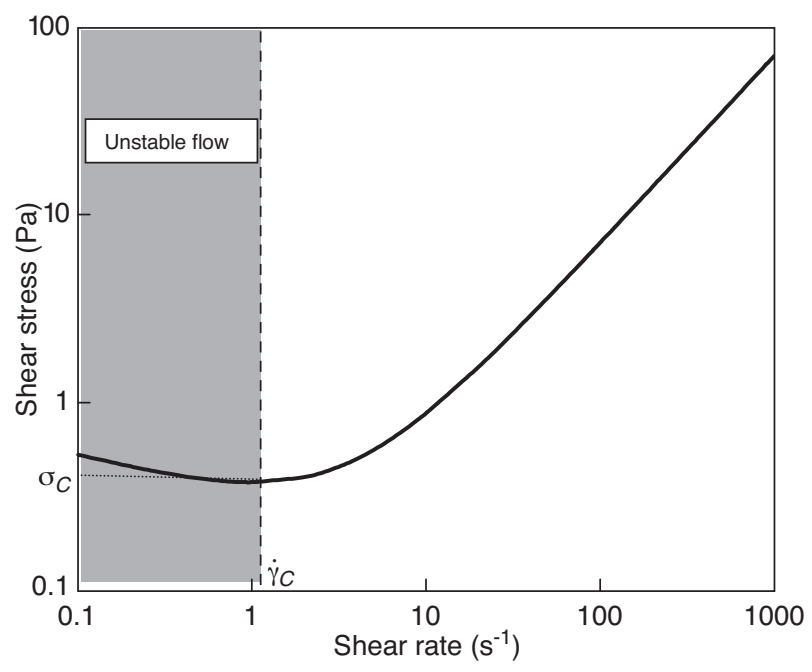

Figure 6

Steady State behavior of a virtual fluid.

parameters. As a particular consequence it is not possible to realistically describe the solid-liquid transition for a material initially at rest from such a model. In order to solve this problem we propose to consider the following situation: the material is initially at rest with a state of structure $\lambda_{0}$ and we impose a constant stress $\sigma$. The model predicts that when $\sigma$ is smaller than a critical value $\left(\sigma_{0}\right) \lambda$ continuously increases in time (since, from (2), $d \lambda / d t>0$ ) and tends to infinity (because $d^{2} \lambda / d t^{2}>0$ ) and the apparent viscosity tends to infinity so that the material apparently stops flowing. On the contrary, when $\sigma$ is larger than $\sigma_{0}$ the material evolves towards a steady-state flow at a finite rate. This critical stress value depends on $\lambda_{0}$ :

$$
\sigma_{0}\left(\lambda_{0}\right)=\frac{\mu_{0}\left(1+\lambda_{0}{ }^{n}\right)}{\alpha \theta \lambda_{0}} \approx \frac{\mu_{0}}{\alpha \theta} \lambda_{0}{ }^{n-1}
$$

This critical stress is thus a kind of apparent yield stress of the material since for a smaller stress the material may slightly deform then stops flowing whereas for a larger stress it goes on flowing indefinitely.

\subsection{Solid-liquid Transition After a Rest}

Let us now consider that the material has been presheared at a high shear rate then left at rest during a time $\Delta t$. At the end of the preshear the value of the structure parameter $\left(\lambda_{i}\right)$ is low $(<<1)$. Then the material restructures according to Equation (2) with $\dot{\gamma}=0$ so that at the end of the rest period we have $\lambda=$ $\lambda_{i}+\Delta t / \theta$ which may be approximated by $\Delta t / \theta$ as soon as $\Delta t>\theta$. Thus the apparent yield stress after a strong preshear and a rest period is:

$$
\sigma_{0}(\Delta t) \approx \frac{\mu_{0}}{\alpha \theta}\left(\frac{\Delta t}{\theta}\right)^{n-1}
$$

\subsection{Transient Behavior Under Controlled Shear Rate}

Let us consider that we suddenly impose a shear rate $\dot{\gamma}$ to a material in the initial state of structure $\lambda_{0}$. By solving the differential Equation (2) we find that the state of the material then evolves from $\lambda_{0}$ to the steady state value $\lambda_{\infty}=1 / \alpha \theta \dot{\gamma}$ according to:

$$
\lambda=\lambda_{\infty}+\left(\lambda_{0}-\lambda_{\infty}\right) \exp -\alpha \dot{\gamma} \mathrm{t}
$$

The shear stress, which in general may be approximated by $\mu_{0}(1+\lambda) \dot{\gamma}$ since $n$ is often close to 1 , also undergoes an exponential decrease from its maximum value to its steadystate value with a characteristic time $1 / \alpha \dot{\gamma}$.

\subsection{Practical Determination of the Parameters of the Model from a Two "Peak Flow Tests"}

We consider the following test: we strongly preshear the material then leave it at rest during a time $\Delta t_{0}$ and then impose a low shear rate $\dot{\gamma}$ while recording the stress evolution in time until reaching an apparent steady-state; we then start again the experiment with now a time of rest $\Delta t_{1}$. These tests are similar to classical normalized tests used in the petroleum industry (API Gel 0 and Gel 10). The corresponding stress curve in time has the typical shape described in 3.5: there is first a rapid increase towards a maximum value $\left(\sigma_{0}\right.$ or $\left.\sigma_{1}\right)$, followed by a relatively slow stress decrease towards a plateau at a level $\sigma_{\infty}$. During the initial stress increase the material is likely in its solid regime, and then it falls in its liquid regime and undergoes a stress decrease. As explained above the state of structure at the solid-liquid transition can hardly be described relevantly. As a consequence we directly assume that the stress peak corresponds to the apparent yield stress under the state of structure obtained after the rest. Under these conditions, using Equation (5) we can deduce the value of the parameter $n$ :

$$
n=1+\frac{\ln \left(\sigma_{1} / \sigma_{0}\right)}{\ln \left(\Delta t_{1} / \Delta t_{0}\right)}
$$

Assuming that the stress curve decreases with a timing similar to that of the state of structure during a typical transient flow (cf. Eq. 7) we deduce that the stress approximately reaches the value $\left(\sigma_{0}+\sigma_{\infty}\right) / 2$ at the time $\Delta_{1 / 2}=1 \mathrm{n} 2 / \alpha \dot{\gamma}$, from which we deduce the second parameter:

$$
\alpha=\frac{\ln 2}{\dot{\gamma} \Delta_{1 / 2}}
$$

Then, using the expression for $\theta$ found from Equation (6) in the steady state expression $\left(\sigma_{\infty}\right)$ as found from Equation (3) we get:

$$
\mu_{0}=\frac{\sigma_{\infty}}{\dot{\gamma}}-\left(\frac{1}{\alpha \dot{\gamma}}\right)^{n} \frac{\alpha \sigma_{1}}{\Delta t_{1}{ }^{n-1}}
$$

and

$$
\theta=\left(\frac{\mu_{0}}{\alpha \sigma_{1}}\right)^{1 / n} \Delta t_{1}^{1-1 / n}
$$




\subsection{Determination of the Model Parameters}

In order to have a better precision on these measurements, we performed the two "peak flow tests" with the help of the Haake RS 150: after having presheared the fluid during 10 minutes at $1000 \mathrm{~s}^{-1}$, the sample was left at rest during a time $\Delta t_{0}=10 \mathrm{~s}$, and a shear rate of $5.1 \mathrm{~s}^{-1}$ is then applied. We then recorded the shear stress $v s$. time curve from which we could estimate more precisely the peak value $\sigma_{0}=5.8 \mathrm{~Pa}$. The same procedure is applied with a rest time $\Delta t_{1}=600 \mathrm{~s}$ and yielded a value $\sigma_{1}=14 \mathrm{~Pa}$. From this curve we can also estimate the time $\Delta_{1 / 2}$ at which the stress reaches the value $\left(\sigma_{1}+\sigma_{\infty}\right) / 2: \Delta_{1 / 2}=0.6 \mathrm{~s}$.

From these measurements, we deduce the values of the different parameters of the model from Equations (8-11) (see Tab.2):

TABLE 2

Experimentallly determined parameters of the model

\begin{tabular}{c|c}
\hline$n$ & 1.215 \\
\hline$\alpha$ & 0.227 \\
\hline$\mu_{0}(\mathrm{~Pa} . \mathrm{s})$ & 0.073 \\
\hline$\theta(\mathrm{s})$ & 0.140 \\
\hline
\end{tabular}

We now have four parameters from which we can describe all the features of the rheological behavior of the fluid, including time-dependent phenomena.

\subsection{Solid Liquid Transition}

Let us first examine the behavior of the fluid after a strong preshear, a given time of rest and then a constant applied shear rate of $5.1 \mathrm{~s}^{-1}$. Measurements of the apparent yield stress were carried out with various times of rest, from 10 seconds (corresponding to Gel 0 procedure) to 900 seconds, using grooved cylinder and a vane geometry.

In Figure 7 are represented the measured apparent yield stresses, i.e. the maximum stress overshoot attained as a function of the time of rest. This "gel value" corresponds to the solid-liquid transition after rest, and is estimated by the model via Equation (6). The results show the good accuracy of the model to represent the thixotropic character of the drilling mud.

One of the simplest rheological features of the behavior of a drilling mud is its steady state shear behavior. As mentioned in the introduction, conventional yield stress shear thinning models are generally used to describe it. The model described here predicts that there exists a critical shear rate under which shear localization appears and no stable flows are possible at smaller shear rates. Correspondingly this critical shear rate is associated with a critical shear stress. From Equation (4) we can deduce this critical stress which can be compared to the value obtained from the measurement in

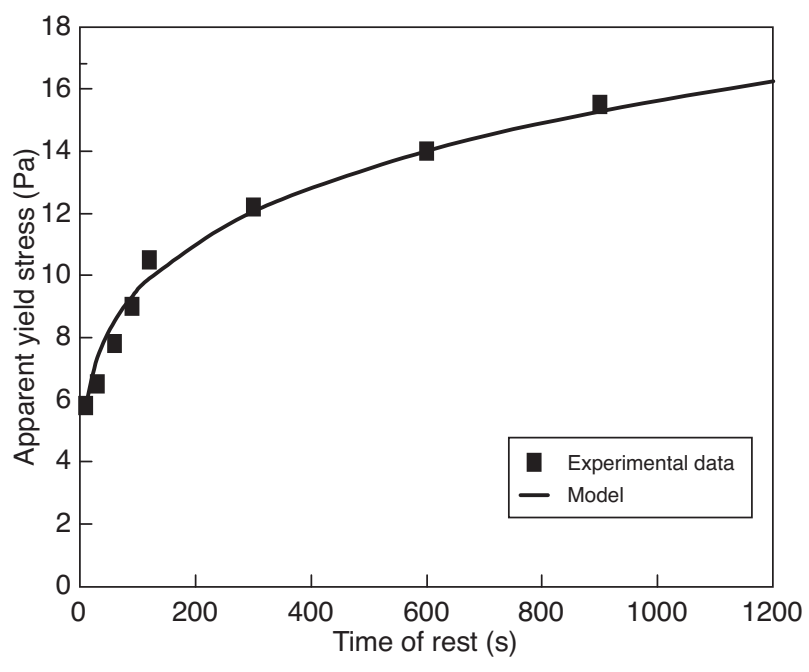

Figure 7

Apparent yield stress as a function of time of rest.

classical rheometry. The model, using parameters values deduced from two simple experiments, gives a value of 3.7 $\mathrm{Pa}$ to compare with the experimentally obtained critical shear stress of $2.85 \mathrm{~Pa}$.

From a physical description of the mechanisms ruling the fluid viscosity (i.e. structuration with time opposed to shear rejuvenation), this simple model is then able to describe steady shear rheology as well as transient behaviour linked to thixotropy.

\subsection{Pressure Peak for the Start Flow of a Complex Fluid in a Conduit}

These characteristics have a strong impact on the phenomena taking place at very low shear rates. One can estimate for example a practical case which is the pressure peak that has to be overcome in order to restart pumping after a static period when drilling a well.

We consider a thixotropic fluid which is flowing at a relatively rapid rate through a concentric conduit of length $\Delta L$ and of internal radius $R_{i}$ and external radius $R_{e}$. We will define an equivalent radius $R$ so that the flow rate and pressure drop for the concentric tube is equivalent to flow rate and pressure drop in a single tube of radius $R$. Analytical expressions for this equivalent diameter may be derived from assumptions on the rheological behavior of the fluid (Reed and Pliehvari, 1993).

The fluid is flowing initially at a relatively large flow rate (high shear) for sufficient time so that we can assume that its state of structure is close to zero. Then, we suppose a stop of circulation: the fluid stops flowing at the initial time and is left at rest during a time $\Delta t$. At that time the circulation is 
resumed and the fluid is moved again at a given rate. In that case, similarly to the above described rheometrical test, the annular pressure drop will first rapidly reach a value $\Delta P$ then progressively decreases to its steady state value (in case of large $\Delta t$ ). This value $\Delta P$ due to aging at rest may be predicted as soon as the rheological parameters of the above model have been determined. Indeed neglecting inertia effects the shear stress distribution is found from the momentum equation and we deduce the expression for the shear stress at the wall:

$$
\sigma_{w}=\frac{\Delta P}{\Delta L} \frac{R}{2}
$$

After a time of rest $\Delta t$, the apparent yield stress of the fluid is given by Equation (6). We can then deduce the initial pressure drop when the fluid start flowing:

$$
\Delta P=\frac{2 \mu_{0}}{\alpha \theta R}\left(\frac{\Delta t}{\theta}\right)^{n-1} \Delta L
$$

This value may be compared to the steady state value of the annular pressure drop for a given flow rate $Q$, in that case for approximate calculation, the Newtonian shear rate at the wall is given by:

$$
\dot{\gamma}=\frac{8 u}{2 R}=\frac{4 Q}{\pi R^{3}}
$$

The steady shear stress at the wall is given by Equation (3), we obtain this way the steady state pressure drop:

$$
\frac{\Delta P_{S}}{\Delta L}=\frac{8 \mu_{0} Q}{\pi R^{4}}\left[1+\left(\frac{\pi R^{3}}{4 \alpha \theta Q}\right)^{n}\right]
$$

An example of approximate calculations is given in Table 3 for a given equivalent diameter of $0.051 \mathrm{~m}$ and a given flow rate of $1500 \mathrm{~L} / \mathrm{min}$.

TABLE 3

Example of calculation of initial pressure drop due to aging

\begin{tabular}{c|c|c}
\hline $\begin{array}{c}\text { Time of rest } \\
\text { (hours) }\end{array}$ & $\begin{array}{c}\text { Initial pressure } \\
\text { drop }(\mathrm{Pa} / \mathrm{m})\end{array}$ & $\begin{array}{c}\text { Steady state } \\
\text { pressure drop }(\mathrm{Pa} / \mathrm{m})\end{array}$ \\
\hline 0.5 & 700 & 770 \\
\hline 1 & 813 & 770 \\
\hline 12 & 1388 & 770 \\
\hline 24 & 1611 & 770 \\
\hline
\end{tabular}

This rough estimation shows how the aging process could be significant in pressure drop estimation. The qualitative trends show that the pressure peak after 12 hours of rest could attain values that are twice the steady state value. When transient phenomena are not taken into account, the initial pressure peak is not considered in the hydraulic calculation. A physical model like the simple one presented here allows to consider both transient and steady state characteristics of the fluid, and therefore improves practical hydraulic considerations.

\section{CONCLUSION}

The flow of complex systems as mixture of emulsions and colloidal suspensions is often characterized by specific behaviors at low deformation, that are the signature of their "jammed" character. Such an industrial complex system (an invert emulsion with organophilic clays) has been studied with a particular focus on the solid liquid transition and its time dependence. Classical stress increasing-decreasing ramp experiments show a peculiar trend at low shear, that can be explained through systematic creep tests and MRI velocimetry. This fluid presents a viscosity bifurcation characterized by a critical shear stress. When a stress is applied after pre shearing, the material will first flow homogeneously on a very long transient period. Then, if the applied stress value is higher than the critical shear stress, the material will reach a steady flow behavior at a shear rate larger than a minimum value. On the other side, when the applied stress is lower than the critical shear stress, the material will progressively increase its viscosity upon complete stoppage. In a geometry where the stress distribution is not homogeneous, this yields "true shear banding" with discontinuous transition between "solid" and liquid phase. This behavior can be explained by structural considerations regarding competition between aging (due to progressive linkage between droplets due to colloidal particles (Ragouilliaux et al., 2007)) and shear rejuvenation.

In the last part of this work, we have tried to propose a unified description of yielding and thixotropy from a single rheological model able to render the observed behavior of the fluid. This rather simple model relies on relevant physical description of the mechanisms that govern the structure and the deformation of such pasty materials. With a limited number of parameters that have all a physical meaning, the model is shown to be able to describe steady state as well as transient rheological properties of the fluid, without need to define a priori values for macroscopic characteristics of the fluid like apparent yield stress or gel building. A pragmatic method to estimate the parameters is proposed, and the model is validated through laboratory measurements. The model predicts unusual behavior for the fluid at very low shear rate like shear localization, which could explain the difficulties associated with such measurements. Finally, we have shown how this model could be used to predict practical transient phenomena like pressure peak after restart.

\section{REFERENCES}

Barnes H.A. (1994) Rheology of emulsions - A review, Colloid. Surface. A 91,89

Bécu L., Grondin P., Colin A, Manneville S. (2004) How does a concentrated emulsion flow? Yielding, local rheology, and wall slip, Colloid. Surface. A 263, 146. 
Bécu L., Manneville S., Colin A. (2006) Yielding and flow in adhesive and nonadhesive concentrated emulsions, Phys. Rev. Lett. 96, 138302.

Cheng D.C.-H., Evans F. (1965) Phenomenological characterization of the rheological behaviour of inelastic reversible thixotropic and antithixotropic fluids, Brit. J. Appl. Phys. 16, 1599-1617.

Chan Man Fong C.F., Turcotte G., De Kee D. (1996) Modelling steady and transient rheological properties, J. Food Eng. 27, 6370.

Coussot P., Leonov A.I., Piau J.M. (1993) Rheology of concentrated dispersed systems in a low molecular weight matrix, J. NonNewton. Fluid 46, 179-217.

Coussot P. (1997) Mudflow rheology and dynamics, Balkema, Rotterdam, Netherlands.

Coussot P., Nguyen Q.D., Huynh H.T., Bonn D. (2002a) Avalanche behavior in yield stress fluids, Phys. Rev. Lett. 88, 175501.

Coussot P., Nguyen Q.D., Huynh H.T., Bonn D. (2002b) Viscosity bifurcation in thixotropic, yielding fluids, J. Rheol. 46, 573-589.

Coussot P., Roussel N., Jarny S., Chanson H. (2005) Catastrophic or continuous solid-liquid transition in jammed systems, Phys. Fluids 17, 011704 .

Coussot P. (2005) Rheometry of pastes, suspensions and granular materials - Application in industry and environnement, Wiley, New York.

De Kee D., Chan Man Fong C.F. (1994) Rheological properties of structured fluids, Polym. Eng. Sci. 34, 438-445.

Herzhaft B., Rousseau L., Néau L., Moan M., Bossard F. (2003) Influence of temperature and clays/emulsion microstructure on oilbased mud low shear rate rheology, SPE J. 8, 3, 211-217.

Hollingsworth K.G., Johns M.L. (2004) Rheo-nuclear magnetic resonance of emulsion systems, J. Rheol. 48, 787.
Mason T.G., Bibette J., Weitz D.A. (1995) Elasticity of compressed emulsions, Phys. Rev. Lett. 75, 2051-2054.

Mason T.G., Bibette J., Weitz D.A. (1996) Yielding and flow of monodisperse emulsions, J. Colloid Interf. Sci. 179, 439.

Mujumdar A., Beris A., Metzner A. (2001) Transient phenomena in thixotropic systems, J. Non-Newton. Fluid 2072, 1-22.

Ragouillaux A., Herzhaft B., Bertrand F., Coussot P. (2006) Flow instability and shear localization in a drilling emulsion, Rheol. Acta 46, 2, 261-271.

Ragouilliaux A., Ovarlez G., Shaidzdeh-Bonn N., Herzhaft B., Palermo T., Coussot P. (2007) Transition from a simple yield-stress fluid to a thixotropic material, Phys. Rev. E 76, 051408.

Raynaud J.S., Moucheront P., Baudez J.C., Bertrand F., Guilbaud J.P., Coussot P. (2002) Direct determination by nuclear magnetic resonance of the thixotropic and yielding behavior of suspensions, $J$. Rheol. 46, 709.

Reed T., Pliehvari A. (1993) A New Model for Laminar, Transitional, and Turbulent Flow of Drilling Muds, SPE paper 25456 presented at the Production Operation Symposium, Oklahoma City, USA.

Roussel N., Le Roy R., Coussot P. (2004) Test of a thixotropy model by comparison with local and macroscopic flow properties, $J$. Non-Newton. Fluid 117, 85-95.

Yan N., Gray M.R., Masliyah J.H. (2001) On water-in-oil emulsions stabilized by fine solids, Colloid Surface. A 193, 97.

Final manuscript received in March 2009 Published online in September 2009 or distributed for profit or commercial advantage and that copies bear this notice and the full citation on the first page. Copyrights for components of this work owned by others than IFP must be honored. Abstracting with credit is permitted. To copy otherwise, to republish, to post on servers, or to redistribute to lists, requires prior specific permission and/or a fee: Request permission from Documentation, Institut français du pétrole, fax. +33147527078 , or revueogst@ifp.fr. 